\title{
Generalization of the Analytical Exponential Model for Homogeneous Reactor Kinetics Equations
}

\author{
Abdallah A. Nahla ${ }^{1,2}$ and Mohammed F. Al-Ghamdi ${ }^{2}$ \\ ${ }^{1}$ Department of Mathematics, Faculty of Science, Tanta University, Tanta 31527, Egypt \\ ${ }^{2}$ Department of Mathematics, Faculty of Science, Taif University, Taif 888, Saudi Arabia \\ Correspondence should be addressed to Abdallah A. Nahla, nahla@tu.edu.eg
}

Received 24 January 2012; Revised 29 February 2012; Accepted 7 March 2012

Academic Editor: Pablo González-Vera

Copyright (c) 2012 A. A. Nahla and M. F. Al-Ghamdi. This is an open access article distributed under the Creative Commons Attribution License, which permits unrestricted use, distribution, and reproduction in any medium, provided the original work is properly cited.

\begin{abstract}
Mathematical form for two energy groups of three-dimensional homogeneous reactor kinetics equations and average one group of the precursor concentration of delayed neutrons is presented. This mathematical form is called "two energy groups of the point kinetics equations." We rewrite two energy groups of the point kinetics equations in the matrix form. Generalization of the analytical exponential model (GAEM) is developed for solving two energy groups of the point kinetics equations. The GAEM is based on the eigenvalues and the corresponding eigenvectors of the coefficient matrix. The eigenvalues of the coefficient matrix are calculated numerically using visual FORTRAN code, based on Laguerre's method, to calculate the roots of an algebraic equation with real coefficients. The eigenvectors of the coefficient matrix are calculated analytically. The results of the GAEM are compared with the traditional methods. These comparisons substantiate the accuracy of the results of the GAEM. In addition, the GAEM is faster than the traditional methods.
\end{abstract}

\section{Introduction}

In real nuclear reactor problems that involve safety considerations, the coefficients of the space-time neutron diffusion equations depend upon the neutron flux density, precursor concentration of delayed neutrons groups, space, time, and temperature feedback. The numerical methods and the analytical approximations for solving the space-time neutron diffusion equations in the nuclear reactor have been of interest in nuclear physics and reactor design.

Three-dimensional nodal mixed dual method was used for the nuclear reactor kinetics equations with improved quasistatic model and the semiimplicit scheme to solve the precursor equations [1]. A rigorous weight function in the quasistatic method was presented to solve the time-dependent multigroup diffusion equations for subcritical systems with external sources [2]. Parallelised Krylov methods were applied to the improved quasistatic approach in addition to the direct, implicit time difference, approach for solving 
space-time dependent multigroup neutron diffusion equations [3]. The nodal diffusion method was presented to solve space-time neutron kinetics using the finite element, primal and mixed hybrid nodal methods [4]. A one-step implicit method and a nodal modal method were studied for the solution of the time-dependent neutron diffusion equations which-based on a hexagonal spatial mesh [5-7]. An adiabatic kinetics model was developed for a boiling water reactor (BWR) core simulator AETNA [8]. Mathematical modeling of the space-time kinetics phenomena in advanced heavy water reactor, a $920 \mathrm{MW}$ thermal, vertical pressure tube type thorium-based nuclear reactor, was presented using nodal modal method [9]. Class of Padé and cut-product approximations were applied to solve the two energy groups space-time nuclear reactor kinetics equations in two dimensions [10]. Adaptive Matrix Formation (AMF) method was presented and applied to homogeneous, symmetric heterogeneous, and nonsymmetric heterogeneous reactors in the cases of two and three dimensions [11]. The generalized Runge-Kutta method was developed for solving the multigroup, multidimensional, static, and transient neutron diffusion kinetics equations [12]. Computation accuracy and efficiency of a power series analytic method were presented for the time-space-dependent neutron diffusion equations with adiabatic heat up and doppler feedback [13]. The multigroup integro-differential equations of the neutron diffusion kinetics were presented and solved numerically in multislab geometry with the use of the progressive polynomial approximation [14].

The different kinds of Padé approximations were applied to the nonlinear point kinetics equations including temperature feedback via analytical inversion method $[15,16]$. The straightforward recurrence relation of a power series was presented to solve the point kinetics equations in the presence of delayed neutrons with Newtonian feedback [17]. The stability of generalized Runge-Kutta method of order four was studied for the stiff system point kinetics equations with temperature feedback reactivity $[18,19]$. The analytical exponential model was generalized to solve the point kinetics equations of beryllium and heavy water moderated reactors with step, ramp, and temperature feedback reactivities [20]. Power series solution (PWS) was applied for solving the point kinetics equations with lumped model temperature feedback [21]. Recently, new analytical solution, based on the roots of inhour equation, the eigenvalues of the coefficient matrix, was presented and applied for the point kinetics equations with the constant, ramp, and temperature feedback reactivities [22].

In this work, the two energy groups of the point kinetics equations and the average one group of delayed neutron are introduced. The generalization of the analytical exponential model is calculated using the eigenvalues and the eigenvectors of the coefficient matrix. The eigenvalues and the corresponding eigenvectors of the coefficient matrix are calculated. The results of the generalization of the analytical exponential model are compared with the results of the traditional methods.

\section{Mathematical Model}

The two energy groups neutron diffusion equations governing the dynamic groups diffusion neutron flux and average one group of the precursor concentration of delayed neutrons behavior are written as follows [23-25]:

$$
\begin{aligned}
\frac{1}{v_{1}} \frac{\partial}{\partial t} \Phi_{1}(r, t)= & D_{1} \nabla^{2} \Phi_{1}(r, t)-\left(\Sigma_{a_{1}}+\Sigma_{S_{12}}\right) \Phi_{1}(r, t)+v \Sigma_{f_{1}}(1-\beta) \Phi_{1}(r, t) \\
& +v \Sigma_{f_{2}}(1-\beta) \Phi_{2}(r, t)+\lambda C(r, t),
\end{aligned}
$$




$$
\begin{aligned}
\frac{1}{v_{2}} \frac{\partial}{\partial t} \Phi_{2}(r, t) & =D_{2} \nabla^{2} \Phi_{2}(r, t)-\Sigma_{a_{2}} \Phi_{2}(r, t)+\Sigma_{s_{12}} \Phi_{1}(r, t), \\
\frac{\partial}{\partial t} C(r, t) & =\beta\left(v \Sigma_{f_{1}} \Phi_{1}(r, t)+v \Sigma_{f_{2}} \Phi_{2}(r, t)\right)-\lambda C(r, t),
\end{aligned}
$$

where $\Phi_{1}(r, t)$ and $\Phi_{2}(r, t)$ are fast and thermal neutron flux, $C(r, t)$ is average one group of the precursor concentration of delayed neutrons, $D_{1}$ and $D_{2}$ are fast and thermal diffusion coefficients, $\Sigma_{a_{1}}$ and $\Sigma_{a_{2}}$ are fast and thermal absorption cross-sections, $\Sigma_{f_{1}}$ and $\Sigma_{f_{2}}$ are fast and thermal fission cross-sections, $\Sigma_{s_{12}}$ is the scattering cross-section from fast-to-thermal neutron, $v$ is the neutron fission, $v_{1}$ and $v_{2}$ are fast and thermal neutron speed, $\lambda$ is decay constant of average one group of delayed neutrons, and $\beta$ is the fraction of delayed neutrons.

The neutron flux and the precursor concentration of delayed neutrons can be written as separable functions of space and time as

$$
\begin{gathered}
\Phi_{1}(r, t)=v_{1} n_{1}(t) \widehat{\Psi}(r), \\
\Phi_{2}(r, t)=v_{2} n_{2}(t) \widehat{\Psi}(r), \\
C(r, t)=c(t) \widehat{\Psi}(r),
\end{gathered}
$$

where $n_{1}(t)$ and $n_{2}(t)$ are the fast and thermal neutron density, $c(t)$ is the precursor concentration density of delayed neutrons and $\widehat{\Psi}(r)$ is the fundamental function which can be determined from the following:

$$
\nabla^{2} \widehat{\Psi}(r)+B^{2} \widehat{\Psi}(r)=0
$$

where $B^{2}$ is the material buckling.

Substituting (2.2) and (2.3) into (2.1) yields

$$
\begin{aligned}
\frac{d n_{1}(t)}{d t}= & -D_{1} B^{2} v_{1} n_{1}(t)-\left(\Sigma_{a_{1}}+\Sigma_{s_{12}}\right) v_{1} n_{1}(t)+v \Sigma_{f_{1}}(1-\beta) v_{1} n_{1}(t) \\
& +v \Sigma_{f_{2}}(1-\beta) v_{2} n_{2}(t)+\lambda c(t) \\
\frac{d n_{2}(t)}{d t}= & -D_{2} B^{2} v_{2} n_{2}(t)-\Sigma_{a_{2}} v_{2} n_{2}(t)+\Sigma_{s_{12}} v_{1} n_{1}(t) \\
\frac{d c(t)}{d t}= & \beta\left(v \Sigma_{f_{1}} v_{1} n_{1}(t)+v \Sigma_{f_{2}} v_{2} n_{2}(t)\right)-\lambda c(t) .
\end{aligned}
$$

Let us consider that $l_{1}=1 / v_{1} v \Sigma_{f_{1}}$ and $l_{2}=1 / v_{2} v \Sigma_{f_{2}}$ are fast and thermal generation time of neutrons, $L_{1}^{2}=D_{1} / \Sigma_{a_{1}}$ and $L_{2}^{2}=D_{2} / \Sigma_{a_{2}}$ are fast and thermal diffusion length, $k_{1}=v \Sigma_{f_{1}} / \Sigma_{a_{1}}\left[1+L_{1}^{2} B^{2}\right]$ and $k_{2}=v \Sigma_{f_{2}} / \Sigma_{a_{2}}\left[1+L_{2}^{2} B^{2}\right]$ are fast and thermal multiplication factor, $\rho_{1}=\left(k_{1}-1\right) / k_{1}$ and $\rho_{2}=\left(k_{2}-1\right) / k_{2}$ are fast and thermal reactivities, $\kappa=v_{1} \Sigma_{s_{12}}$, $\mu_{1}=\beta / l_{1}$ and $\mu_{2}=\beta / l_{2}$. 
Equations (2.4) are rewritten in the following form:

$$
\begin{aligned}
\frac{d n_{1}(t)}{d t} & =\left(\frac{\rho_{1}}{l_{1}}-\mu_{1}-\kappa\right) n_{1}(t)+\left(\frac{1}{l_{2}}-\mu_{2}\right) n_{2}(t)+\lambda_{c}(t), \\
\frac{d n_{2}(t)}{d t} & =\kappa n_{1}(t)+\left(\frac{\rho_{2}-1}{l_{2}}\right) n_{2}(t), \\
\frac{d c(t)}{d t} & =\mu_{1} n_{1}(t)+\mu_{2} n_{2}(t)-\lambda c(t) .
\end{aligned}
$$

Aboanber [26] presented two-energy group two-point kinetic model of reflected reactor. So, (2.5) can be called "two energy groups of the point kinetics equations".

The initial values of this system, $d n_{1}(0) / d t=d n_{2}(0) / d t=d c(0) / d t=0$, take the following form

$$
\begin{gathered}
n_{1}(0)=\frac{\left[1-\rho_{2}\right]}{l_{2} \kappa} n_{2}(0), \\
c(0)=\frac{\mu_{1}}{\lambda} n_{1}(0)+\frac{\mu_{2}}{\lambda} n_{2}(0),
\end{gathered}
$$

and the material buckling takes the form:

$$
\begin{aligned}
B^{2}= & -\frac{\Sigma_{a_{1}}+\Sigma_{S_{12}}-v \Sigma_{f_{1}}}{2 D_{1}}-\frac{\Sigma_{a_{2}}}{2 D_{2}} \\
& +\sqrt{\left(\frac{\Sigma_{a_{1}}+\Sigma_{s_{12}}-v \Sigma_{f_{1}}}{2 D_{1}}+\frac{\Sigma_{a_{2}}}{2 D_{2}}\right)^{2}-\left(\frac{\Sigma_{a_{2}}\left(\Sigma_{a_{1}}+\Sigma_{s_{12}}-v \Sigma_{f_{1}}\right)-\Sigma_{s_{12}} v \Sigma_{f_{2}}}{D_{1} D_{2}}\right)} .
\end{aligned}
$$

\section{Generalization of the Analytical Exponential Model}

Let us rewrite (2.5) in matrix form as follows

$$
\frac{d}{d t} \Psi(t)=\mathbf{A} \Psi(t)
$$

where

$$
\begin{gathered}
\Psi(t)=\left[\begin{array}{l}
n_{1}(t) \\
n_{2}(t) \\
c(t)
\end{array}\right], \quad \mathbf{A}=\left[\begin{array}{ccc}
-\alpha & \zeta & \lambda \\
\kappa & -\eta & 0 \\
\mu_{1} & \mu_{2} & -\lambda
\end{array}\right], \\
\alpha=\mu_{1}+\kappa-\frac{\rho_{1}}{l_{1}}, \quad \zeta=\frac{1}{l_{2}}-\mu_{2}, \quad \eta=\frac{1-\rho_{2}}{l_{2}} .
\end{gathered}
$$


Using the exponential integrator $[27,28]$, we can determine the general solution of $(3.1)$ as follows:

$$
\Psi(t)=B \exp \left(\int_{t_{0}}^{t} \mathbf{A} d t\right)
$$

where $B$ is the integral constant.

This solution includes the exponential function of the matrix A which can be calculated using generalization of the analytical exponential model $[20,29]$ as

$$
\Psi(t)=\sum_{k=1}^{3} B_{k} \exp \left(\omega_{k} t\right) \mathbf{U}_{k}
$$

where $B_{k}$ are the constants, $\omega_{k}$ are the eigenvalues of the matrix $\mathbf{A}$ and $\mathbf{U}_{k}$ are the corresponding eigenvectors of the matrix $\mathbf{A}$.

Using the initial condition $\Psi(0)=\Psi_{0}$ to determine the constants $B_{k}=\mathbf{V}_{k}^{T} \Psi_{0}$, (3.4) becomes as follows

$$
\Psi(t)=\sum_{k=1}^{3} \exp \left(\omega_{k} t\right) \mathbf{U}_{k} \mathbf{V}_{k}^{T} \Psi_{0}
$$

where, $\mathbf{V}_{k}$ are the eigenvectors of matrix $\mathbf{A}^{T}$ which corresponding to eigenvalues $\omega_{k}$ and satisfies the normalization condition $\mathbf{U}_{k}^{T} \mathbf{V}_{k}=1$. The eigenvectors $\mathbf{U}_{k}$ and $\mathbf{V}_{k}$ of the coefficient matrix $\mathbf{A}$ and $\mathbf{A}^{T}$, respectively, are calculated analytically in the next subsections.

Equation (3.5) gives good results only for a constant matrix $\mathbf{A}$. But for variable matrix A, the solution of the equation (3.1) can be takes the form

$$
\Psi\left(t_{m+1}\right)=\sum_{k=1}^{3} \exp \left(h \omega_{k}\right) \mathbf{U}_{k} \mathbf{V}_{k}^{T} \Psi\left(t_{m}\right)
$$

where, the values of the eigenvalues $\omega_{k}$ and the corresponding eigenvectors $\mathbf{U}_{k}$ and $\mathbf{V}_{k}$ are calculated at time $t_{m}+(h / 2)$, and $h=t_{m+1}-t_{m}$ is the time step interval $\left[t_{m}, t_{m+1}\right]$.

Equations (3.5) and (3.6) represent the solutions of the two energy groups of point kinetics equation with average one group of delayed neutrons. These solutions represent a generalization of the analytical exponential model for step and variable coefficient matrix $\mathbf{A}$, respectively.

\subsection{Eigenvalues of the Matrix A}

The eigenvalues of the matrix $\mathbf{A}$ are determined from the following algebraic equation:

$$
\omega^{3}+\omega^{2}(\alpha+\eta+\lambda)+\omega\left[\alpha \eta+\alpha \lambda+\eta \lambda-\kappa \zeta-\lambda \mu_{1}\right]+\alpha \eta \lambda-\kappa \zeta \lambda-\kappa \lambda \mu_{2}-\eta \lambda \mu_{1}=0 .
$$

The equation (3.7) is called "inhour equation." This equation has a 3rd degree algebraic equation. To determine the roots of (3.7), we use the visual FORTRAN code, based on 
Laguerre's numerical method, to calculate the roots of an algebraic equation with real coefficients.

\subsection{Eigenvectors of the Matrix A}

The eigenvectors of the matrix $\mathbf{A}$ are determined analytically as follows:

$$
\mathbf{U}_{k}=\left[\begin{array}{c}
1 \\
\frac{\kappa}{\left(\omega_{k}+\eta\right)} \\
\frac{\mu_{1}\left(\omega_{k}+\eta\right)+\kappa \mu_{2}}{\left(\omega_{k}+\lambda\right)\left(\omega_{k}+\eta\right)}
\end{array}\right] .
$$

\subsection{Eigenvectors of the Matrix $A^{T}$}

The eigenvectors of the matrix $\mathbf{A}^{T}$ are determined analytically as follows:

$$
\begin{aligned}
& \mathbf{V}_{k}=\frac{1}{1+\left(\kappa \zeta\left(\omega_{k}+\lambda\right)+\kappa \lambda \mu_{2}\right) /\left(\omega_{k}+\lambda\right)\left(\omega_{k}+\eta\right)^{2}+\left(\lambda \mu_{1}\left(\omega_{k}+\eta\right)+\kappa \lambda \mu_{2}\right) /\left(\omega_{k}+\lambda\right)^{2}\left(\omega_{k}+\eta\right)} \\
& \times\left[\begin{array}{c}
1 \\
\frac{\zeta\left(\omega_{k}+\lambda\right)+\lambda \mu_{2}}{\left(\omega_{k}+\lambda\right)\left(\omega_{k}+\eta\right)} \\
\frac{\lambda}{\left(\omega_{k}+\lambda\right)}
\end{array}\right]
\end{aligned}
$$

where $\left(1+\left(\kappa \zeta\left(\omega_{k}+\lambda\right)+\kappa \lambda \mu_{2}\right) /\left(\omega_{k}+\lambda\right)\left(\omega_{k}+\eta\right)^{2}+\left(\lambda \mu_{1}\left(\omega_{k}+\eta\right)+\kappa \lambda \mu_{2}\right) /\left(\omega_{k}+\lambda\right)^{2}\left(\omega_{k}+\eta\right)\right)^{-1}$ is the normalization factor.

\section{Results and Discussions}

To check the accuracy of the results of the mathematical model of two energy groups of the homogeneous reactor kinetics equations, it is applied to the three-dimensional homogeneous reactor. This reactor is a bare homogeneous cube of side length $200 \mathrm{~cm}$ on each side with two neutron groups and one precursor group. The parameters of the three-dimensional homogeneous reactor are taken from [11] and shown in Table 1. The mathematical model of two energy groups of the point kinetics equations are solved by the generalization of the analytical exponential model (GAEM). Two different types of problems; step and ramp reactivities, are applied.

\subsection{Step Reactivity}

The thermal neutron flux at the center point of the two energy groups of the homogeneous reactor kinetics equations with a positive step reactivity, $\Delta \Sigma_{a_{2}}=-0.0000369$, is calculated in 
Table 1: Parameters of three-dimensional homogeneous reactor [11].

\begin{tabular}{lcc}
\hline Parameter & Group 1 & Group 2 \\
\hline Diffusion coefficient $D(\mathrm{~cm})$ & 1.35 & 1.08 \\
Absorption cross-section $\Sigma_{a}\left(\mathrm{~cm}^{-1}\right)$ & 0.001382 & 0.0054869 \\
Neutron fission $\boldsymbol{v}$ (neutron) & 2.41 & 2.41 \\
Fission cross-section $\Sigma_{f}\left(\mathrm{~cm}^{-1}\right)$ & 0.000242 & 0.00408 \\
Scattering cross-section $\Sigma_{s_{g, g+1}}\left(\mathrm{~cm}^{-1}\right)$ & 0.0023 & 0.0 \\
Velocity $v(\mathrm{~cm} / \mathrm{s})$ & $3.0 \times 10^{7}$ & $2.2 \times 10^{5}$ \\
\hline Decay constant $\lambda\left(\mathrm{s}^{-1}\right)$ & 0.08 & \\
Fraction of delayed neutron $\beta$ & 0.0064 & \\
$K_{\text {eff }}$ & 0.8952858 & \\
\hline
\end{tabular}

Table 2: Thermal neutron flux at the center point of three-dimensional homogeneous reactor with a positive step reactivity.

\begin{tabular}{lccccr}
\hline \multirow{2}{*}{ Time $(\mathrm{s})$} & 3DKIN & AMF & \multicolumn{2}{c}{ GAEM } & Exact \\
\hline 0.0 & $h=0.001$ & $h=0.0001$ & $h=0.001$ & $h=0.0001$ & \\
0.05 & 0.816 & 0.816356 & 0.816356 & 0.816356 & 0.816 \\
0.10 & 1.124 & 1.127264 & 1.127320 & 1.127300 & 1.127 \\
0.15 & 1.406 & 1.407563 & 1.407587 & 1.407538 & 1.407 \\
0.20 & 1.660 & 1.660798 & 1.660782 & 1.660695 & 1.660 \\
0.30 & 1.890 & 1.890179 & 1.890112 & 1.889979 & 1.890 \\
0.40 & 2.289 & 2.288396 & 2.288189 & 2.287949 & 2.288 \\
& 2.622 & 2.621201 & 2.620796 & 2.620432 & 2.620 \\
\hline
\end{tabular}

Table 2. The results of the GAEM for the two energy groups of the point kinetics equations have an accuracy comparable with the results of the adaptive matrix formation (AMF) [11], 3DKIN [30] and the exact solution for the space-time nuclear reactor kinetics equations.

The fast and thermal neutron flux of the two energy groups of the point kinetics equations with a negative step reactivity, $\Delta \Sigma_{a_{2}}=+0.0000369$, is calculated in Table 3 . This table substantiates the accuracy of the results of the GAEM compared with the results of the AMF [11].

Figure 1. shows the fast and thermal neutron flux at three values for positive step reactivities, $\Delta \Sigma_{a_{2}}=-0.0000369,-2(0.0000369)$ and $-3(0.0000369)$. And, the fast and thermal neutron flux at three types of negative step reactivities, $\Delta \Sigma_{a_{2}}=+0.0000369,+2(0.0000369)$ and $+3(0.0000369)$, are shown in Figure 2.

The calculation times, CPU times, of the GAEM to the AMF method are $0.43 \mathrm{~s}: 0.79 \mathrm{~s}$ at the same time step using personal desktop computer (processor: Core $2 \mathrm{Duo}, 2.93 \mathrm{GHz}$ ). This means that the GAEM for two energy groups of the point kinetics equations is faster than the AMF for the space-time nuclear reactor kinetics equations.

\subsection{Ramp Reactivity}

To substantiate the accuracy and the efficiency of the generalization of the analytical exponential model for solving two energy groups of the point kinetics equations with linear ramp reactivity, it is applied to three dimensional homogeneous reactor. The fast and thermal neutron flux for a positive ramp reactivity, $\Delta \Sigma_{a_{2}}=-(0.0000369 / 0.4) t$, are calculated in 
Table 3: Neutron flux at the center point of three-dimensional homogeneous reactor with a negative step reactivity.

\begin{tabular}{|c|c|c|c|c|}
\hline \multirow{2}{*}{ Time (s) } & \multicolumn{2}{|c|}{$\mathrm{AMF}$} & \multicolumn{2}{|c|}{ GAEM } \\
\hline & Fast & Thermal & Fast & Thermal \\
\hline 0.0 & 2.228929 & 0.816356 & 2.228929 & 0.816356 \\
\hline 0.05 & 1.645481 & 0.601048 & 1.645513 & 0.601060 \\
\hline 0.10 & 1.409437 & 0.513969 & 1.409514 & 0.513998 \\
\hline 0.15 & 1.313217 & 0.478479 & 1.313283 & 0.478504 \\
\hline 0.20 & 1.273214 & 0.463731 & 1.273261 & 0.463748 \\
\hline 0.30 & 1.247508 & 0.454268 & 1.247519 & 0.454272 \\
\hline 0.40 & 1.239679 & 0.451400 & 1.239661 & 0.451394 \\
\hline
\end{tabular}

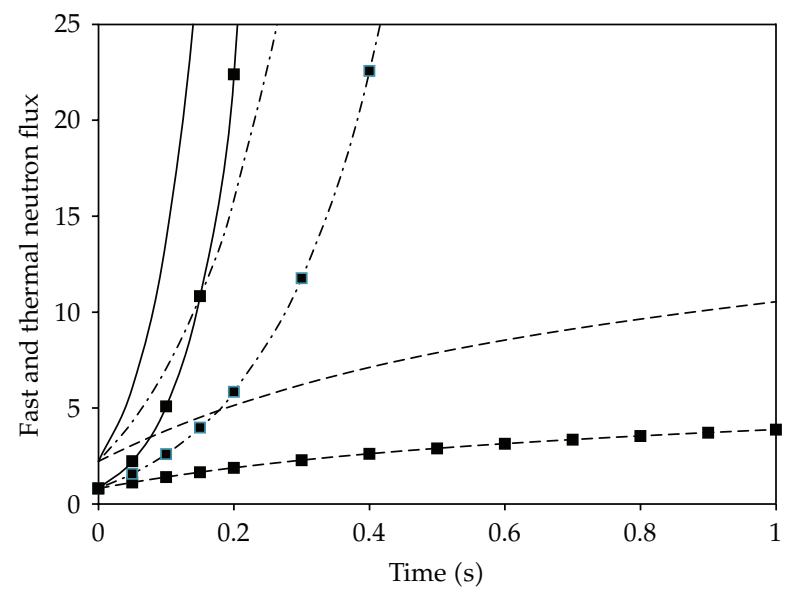

$---(\Delta \Sigma)$ fast neutron flux $\quad-\mathbf{- a}-(\Delta \Sigma)$ thermal neutron flux

...- $(2 \Delta \Sigma)$ fast neutron flux _. - - $-(2 \Delta \Sigma)$ thermal neutron flux

$-(3 \Delta \Sigma)$ fast neutron flux $\rightarrow(3 \Delta \Sigma)$ thermal neutron Flux

Figure 1: Fast and thermal neutron flux at a positive step reactivity.

Table 4. This table is demonstrates the comparison between the results of the GAEM and AMF.

The fast and thermal neutron flux of the two energy groups of the point kinetics equations with a negative ramp reactivity, $\Delta \Sigma_{a_{2}}=+(0.0000369 / 0.4) t$, is calculated in Table 5 .

The fast and thermal neutron flux at different types of positive ramp reactivities, $\Delta \Sigma_{a_{2}}=-(0.0000369 t),-2(0.0000369 t)$ and $-3(0.0000369 t)$, is shown in Figure 3. And also, the fast and thermal neutron flux at different types of negative ramp reactivities, $\Delta \Sigma_{a_{2}}=$ $+(0.0000369 t),+2(0.0000369 t)$ and $+3(0.0000369 t)$, are shown in Figure 4.

For ramp reactivity, The calculation times, CPU times, of the GAEM to the AMF method are $0.45 \mathrm{~s}: 0.81 \mathrm{~s}$. This means, the GAEM is faster than the AMF.

\section{Conclusions}

Two energy groups of the point kinetics equations are represented a mathematical form for three dimensional homogeneous reactor kinetics equations and average one group of 


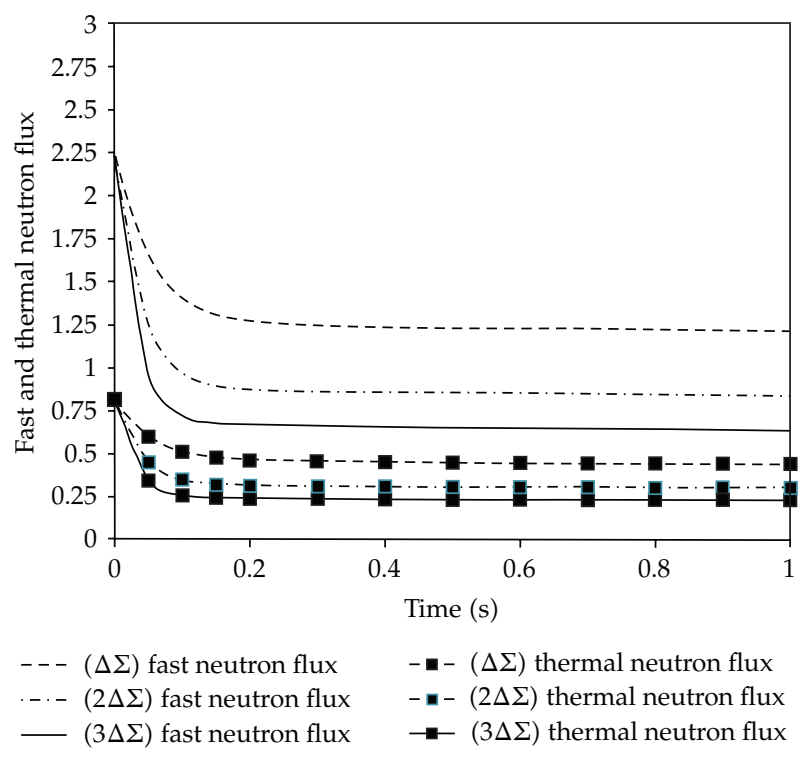

Figure 2: Fast and thermal neutron flux at a negative step reactivity.

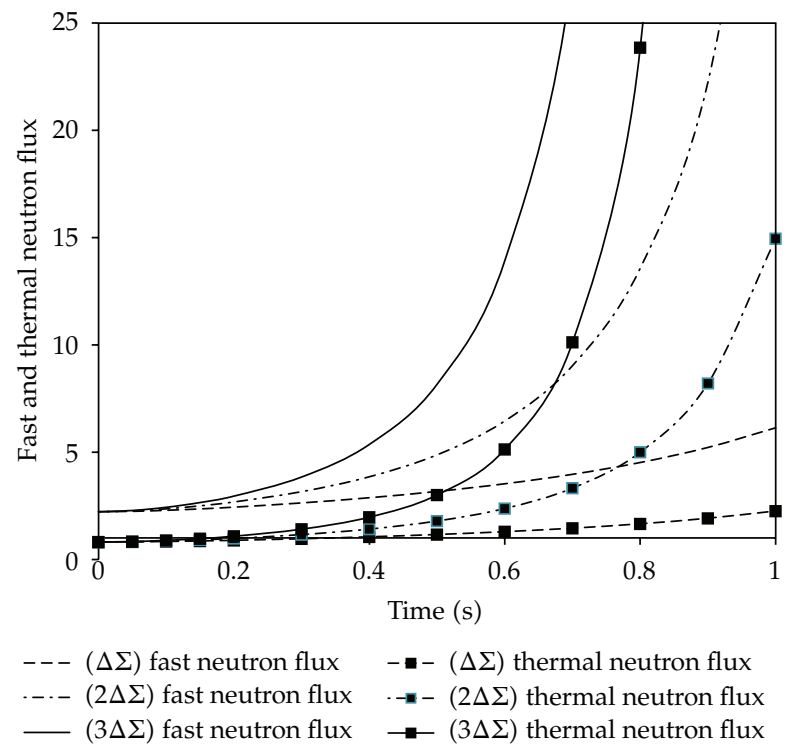

Figure 3: Fast and thermal neutron flux at a positive ramp reactivity.

precursor delayed neutrons. The mathematical form was rewritten in the matrix form. Generalization of the analytical exponential model (GAEM) was developed to solve the new mathematical matrix form for three dimensional homogeneous reactor. This GAEM based on the eigenvalues and the corresponding eigenvectors of the coefficient matrix.

The results of GAEM were compared with the AMF, 3DKIN, and the exact solution for three dimensional homogeneous reactor. This comparison substantiated the accuracy of the results of the GAEM for different types of reactivities. In addition, the GAEM for the 


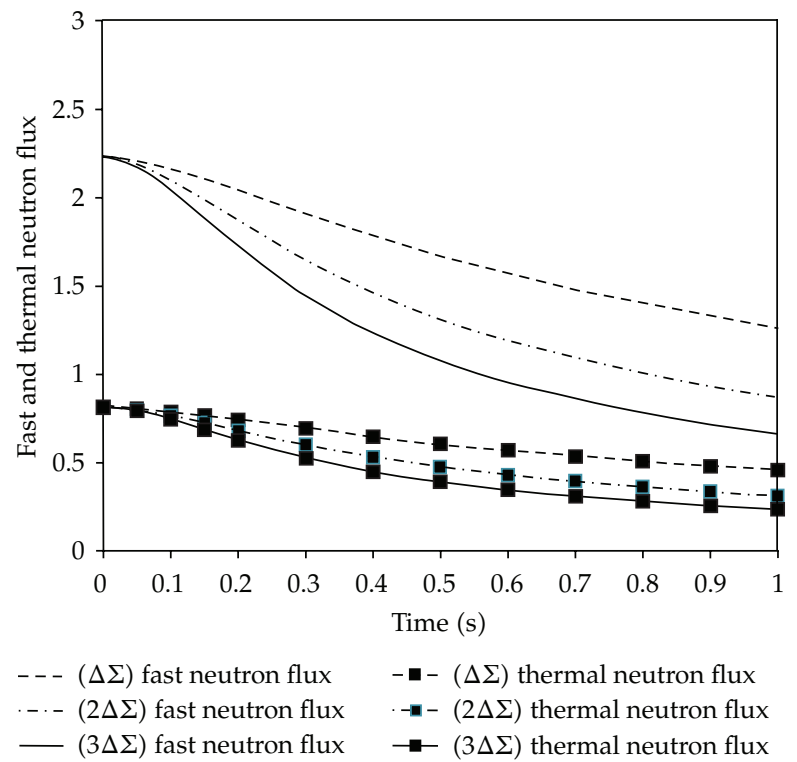

Figure 4: Fast and thermal neutron flux at a negative ramp reactivity.

Table 4: Neutron flux at the center point of three dimensional homogeneous reactor with a positive ramp reactivity.

\begin{tabular}{|c|c|c|c|c|}
\hline \multirow{2}{*}{ Time (s) } & \multicolumn{2}{|c|}{ AMF } & \multicolumn{2}{|c|}{ GAEM } \\
\hline & Fast & Thermal & Fast & Thermal \\
\hline 0.0 & 2.228929 & 0.816356 & 2.228929 & 0.816356 \\
\hline 0.05 & 2.276718 & 0.833995 & 2.276712 & 0.833993 \\
\hline 0.10 & 2.399085 & 0.879151 & 2.399048 & 0.879137 \\
\hline 0.15 & 2.580062 & 0.945931 & 2.579972 & 0.945898 \\
\hline 0.20 & 2.816616 & 1.033217 & 2.816456 & 1.033158 \\
\hline 0.30 & 3.484146 & 1.279522 & 3.483773 & 1.279385 \\
\hline 0.40 & 4.529838 & 1.665354 & 4.529085 & 1.665078 \\
\hline
\end{tabular}

Table 5: Neutron flux at the center point of three dimensional homogeneous reactor with a negative ramp reactivity.

\begin{tabular}{|c|c|c|c|c|}
\hline \multirow{2}{*}{ Time (s) } & \multicolumn{2}{|c|}{ AMF } & \multicolumn{2}{|c|}{ GAEM } \\
\hline & Fast & Thermal & Fast & Thermal \\
\hline 0.0 & 2.228929 & 0.816356 & 2.228929 & 0.816356 \\
\hline 0.05 & 2.182204 & 0.799109 & 2.182170 & 0.799097 \\
\hline 0.10 & 2.072954 & 0.758795 & 2.072892 & 0.758773 \\
\hline 0.15 & 1.936615 & 0.708488 & 1.936528 & 0.708456 \\
\hline 0.20 & 1.795565 & 0.656446 & 1.795460 & 0.656407 \\
\hline 0.30 & 1.541171 & 0.562589 & 1.541033 & 0.562539 \\
\hline 0.40 & 1.340555 & 0.488582 & 1.340396 & 0.488524 \\
\hline
\end{tabular}


mathematical form, two energy groups of the point kinetics equations, is faster than any other traditional methods. So, this mathematical form may be one of the most important models for the neutron diffusion equations in the nuclear reactors.

\section{References}

[1] M. Dahmani, A. M. Baudron, J. J. Lautard, and L. Erradi, “A 3D nodal mixed dual method for nuclear reactor kinetics with improved quasistatic model and a semi-implicit scheme to solve the precursor equations," Annals of Nuclear Energy, vol. 28, no. 8, pp. 805-824, 2001.

[2] K. Kobayashi, "A rigorous weight function for neutron kinetics equations of the quasi-static method for subcritical systems," Annals of Nuclear Energy, vol. 32, no. 8, pp. 763-776, 2005.

[3] A. Gupta, R. S. Modak, H. P. Gupta, V. Kumar, and K. Bhatt, "Parallelised Krylov subspace method for reactor kinetics by IQS approach," Annals of Nuclear Energy, vol. 32, no. 15, pp. 1693-1703, 2005.

[4] L. M. Grossman and J. P. Hennart, "Nodal diffusion methods for space-time neutron kinetics," Progress in Nuclear Energy, vol. 49, no. 3, pp. 181-216, 2007.

[5] D. Ginestar, R. Miro, G. Verdu, and D. Hennig, "A transient modal analysis of a BWR instability event," Journal of Nuclear Science and Technology, vol. 39, no. 5, pp. 554-563, 2002.

[6] R. Miró, D. Ginestar, G. Verdú, and D. Hennig, "A nodal modal method for the neutron diffusion equation. Application to BWR instabilities analysis," Annals of Nuclear Energy, vol. 29, no. 10, pp. 1171-1194, 2002.

[7] S. González-Pintor, D. Ginestar, and G. Verdú, "Time integration of the neutron diffusion equation on hexagonal geometries," Mathematical and Computer Modelling, vol. 52, no. 7-8, pp. 1203-1210, 2010.

[8] M. Tamitani, T. Iwamoto, and B. R. Moore, "Development of kinetics model for BWR core simulator AETNA," Journal of Nuclear Science and Technology, vol. 40, no. 4, pp. 201-212, 2003.

[9] S. R. Shimjith, A. P. Tiwari, M. Naskar, and B. Bandyopadhyay, "Space-time kinetics modeling of Advanced Heavy Water Reactor for control studies," Annals of Nuclear Energy, vol. 37, no. 3, pp. 310324, 2010.

[10] A. E. Aboanber and A. A. Nahla, "Solution of two-dimensional space-time multigroup reactor kinetics equations by generalized Padé and cut-product approximations," Annals of Nuclear Energy, vol. 33, no. 3, pp. 209-222, 2006.

[11] A. E. Aboanber and A. A. Nahla, "Adaptive matrix formation (AMF) method of space-time multigroup reactor kinetics equations in multidimensional model," Annals of Nuclear Energy, vol. 34, no. 1-2, pp. 103-119, 2007.

[12] A. E. Aboanber and Y. M. Hamada, "Generalized Runge-Kutta method for two- and threedimensional space-time diffusion equations with a variable time step," Annals of Nuclear Energy, vol. 35, no. 6, pp. 1024-1040, 2008.

[13] A. E. Aboanber and Y. M. Hamada, "Computation accuracy and efficiency of a power series analytic method for two- and three- space-dependent transient problems," Progress in Nuclear Energy, vol. 51, no. 3, pp. 451-464, 2009.

[14] B. Quintero-Leyva, "The multi-group integro-differential equations of the neutron diffusion kinetics. Solutions with the progressive polynomial approximation in multi-slab geometry," Annals of Nuclear Energy, vol. 37, no. 5, pp. 766-770, 2010.

[15] A. E. Aboanber and A. A. Nahla, "Generalization of the analytical inversion method for the solution of the point kinetics equations," Journal of Physics. A, vol. 35, no. 14, pp. 3245-3263, 2002.

[16] A. E. Aboanber and A. A. Nahla, "Solution of the point kinetics equations in the presence of Newtonian temperature feedback by Padé approximations via the analytical inversion method," Journal of Physics. A, vol. 35, no. 45, pp. 9609-9627, 2002.

[17] A. E. Aboanber and Y. M. Hamada, "Power series solution (PWS) of nuclear reactor dynamics with newtonian temperature feedback," Annals of Nuclear Energy, vol. 30, no. 10, pp. 1111-1122, 2003.

[18] J. Sanchez, "On the numerical solution of the point reactor kinetics equations by generalized RungeKutta methods," Nuclear Science and Engineering, vol. 103, no. 1, pp. 94-99, 1989.

[19] A. E. Aboanber, "Stability of generalized Runge-Kutta methods for stiff kinetics coupled differential equations," Journal of Physics. A, vol. 39, no. 8, pp. 1859-1876, 2006.

[20] A. A. Nahla, "Generalization of the analytical exponential model to solve the point kinetics equations of Be- and D2O-moderated reactors," Nuclear Engineering and Design, vol. 238, no. 10, pp. 2648-2653, 2008. 
[21] T. Sathiyasheela, "Power series solution method for solving point kinetics equations with lumped model temperature and feedback," Annals of Nuclear Energy, vol. 36, no. 2, pp. 246-250, 2009.

[22] A. A. Nahla, "Analytical solution to solve the point reactor kinetics equations," Nuclear Engineering and Design, vol. 240, no. 6, pp. 1622-1629, 2010.

[23] D. L. Hetrick, Dynamics of Nuclear Reactors, American Nuclear Society, La Grange Park, Ill, USA, 1993.

[24] S. Glasstone and A. Sesonske, Nuclear Reactor Engineering, Chapman \& Hall, 1994.

[25] W. M. Stacey, Nuclear Reactor Physics, John Wiley \& Sons, New York, NY, USA, 2001.

[26] A. E. Aboanber, "Spectral effects induced by the presence of a reflector for two-energy group twopoint kinetic model of reflected reactors," Progress in Nuclear Energy, vol. 52, no. 2, pp. 197-205, 2010.

[27] S. Blanes and P. C. Moan, "Fourth- and sixth-order commutator-free Magnus integrators for linear and non-linear dynamical systems," Applied Numerical Mathematics, vol. 56, no. 12, pp. 1519-1537, 2006.

[28] M. Hochbruck and A. Ostermann, "Exponential integrators," Acta Numerica, vol. 19, pp. 209-286, 2010.

[29] A. Quarteroni, R. Sacco, and F. Saleri, Numerical Mathematics, vol. 37 of Texts in Applied Mathematics, Springer, New York, NY, USA, 2000.

[30] D. R. Ferguson and K. F. Hansen, "Solution of the space-dependent reactor kinetics equations in the three dimension," Nuclear Science and Engineering, vol. 51, pp. 189-205, 1973. 


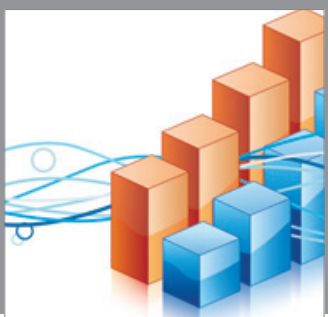

Advances in

Operations Research

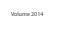

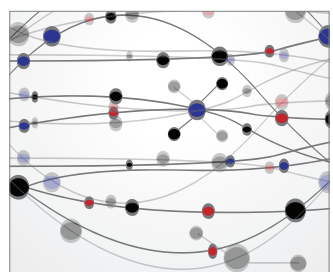

\section{The Scientific} World Journal
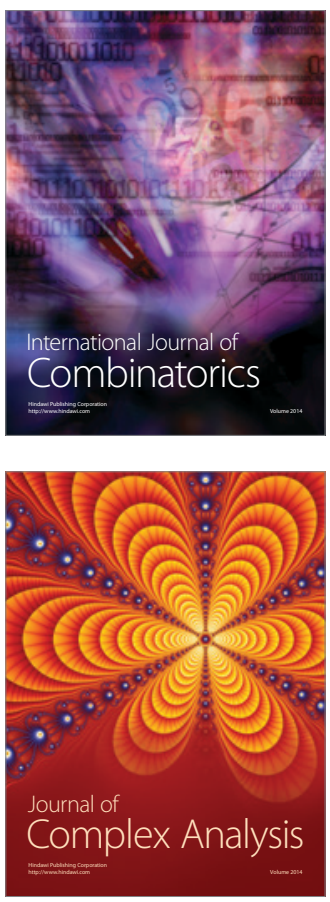

International Journal of

Mathematics and

Mathematical

Sciences
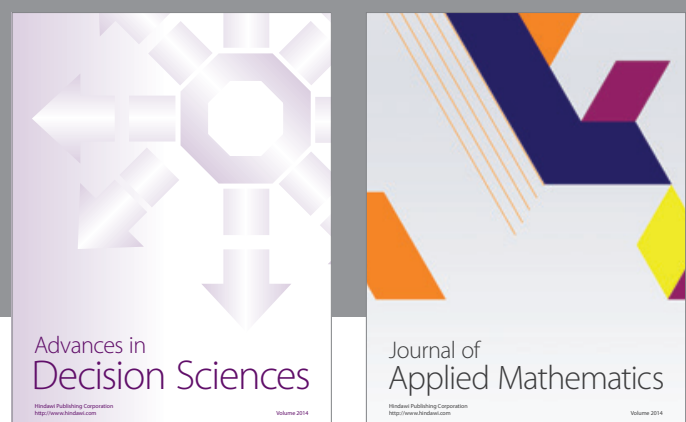

Journal of

Applied Mathematics
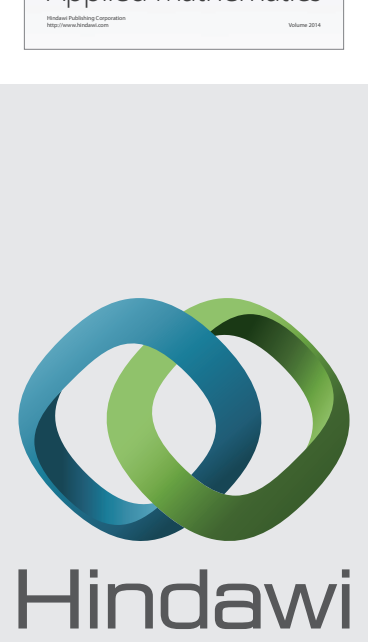

Submit your manuscripts at http://www.hindawi.com
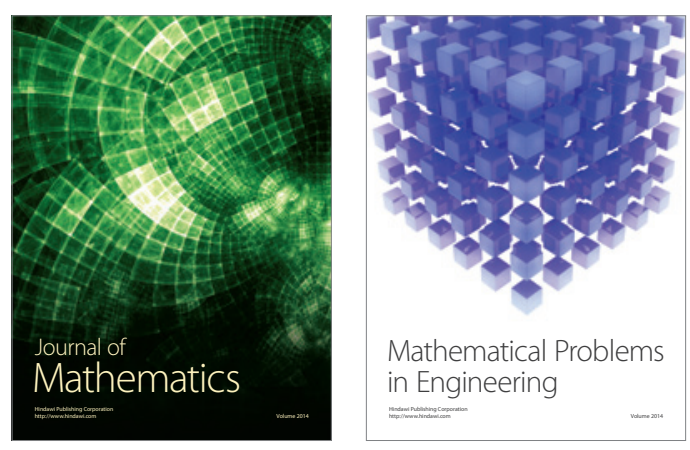

Mathematical Problems in Engineering
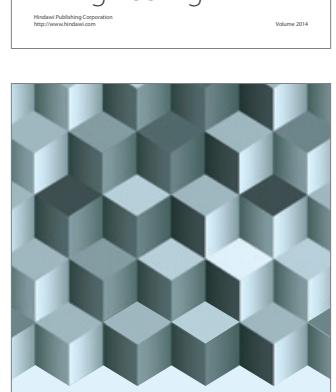

Journal of

Function Spaces
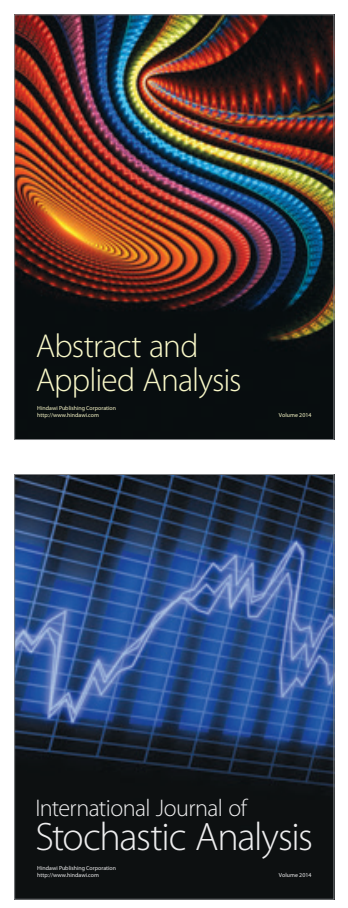

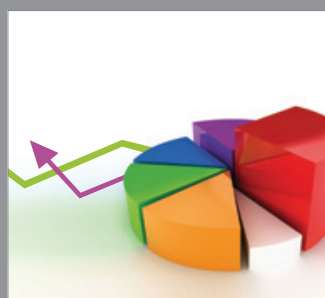

ournal of

Probability and Statistics

Promensencen
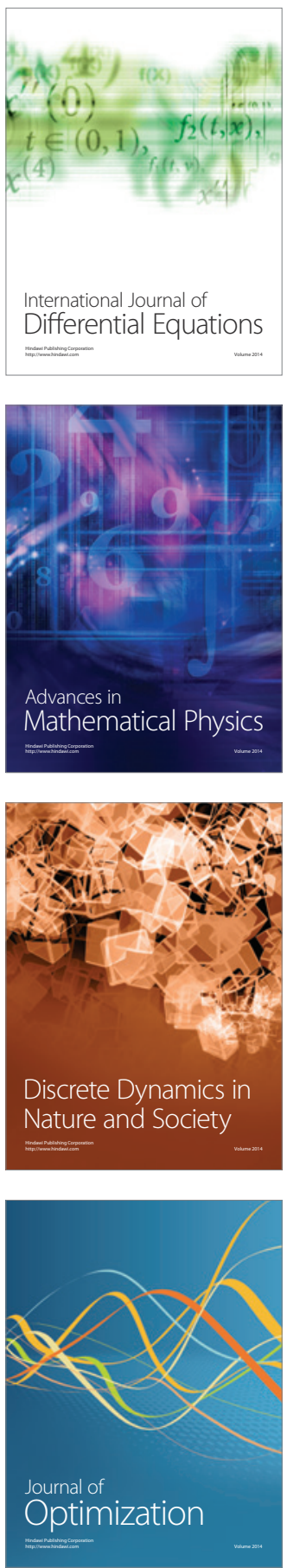